\section{Measuring Reiki Healing - Mystery, Placebo or Real Ener- gy Healing}

\section{Maria Kuman*}

Holistic Research Institute, Knoxville, USA

\begin{abstract}
'Reiki' means 'Universal-Energy' and Reiki Healers, who heal with hands, claim they use universal energy to heal. To prove or disprove their statements, Reiki Healers and their patients were measured with very sensitive equipment before and after treatment. The measurements showed that energy is indeed transferred from the hand healers to the patients, but surprisingly after hand healing not only the energy of the patients will be better balanced and higher, also, the energy of the healers will be higher and better balanced, which proves that the Reiki healers don't use their energy to heal. The measurements show that they channel electric energy through the top of their heads from the atmosphere. However, they could also channel magnetic energy from the earth. The greatest advantage of this type of healing is that, it can be used for prevention. The used equipment could detect energy imbalance 5 to 7 years before the symptoms of the disease would appear. If the energy balance was restored with energy healing or acupuncture, the symptoms of the disease would never appear, and the disease would be prevented.
\end{abstract}

\section{Introduction}

Reiki (Hand) healing was first introduced to the western world by the Buddhist Monk Usui and accepted with skepticism by both, scientists and medical doctors. Are these "healers" doing something by laying their hands on patients or the patients just imagine they are feeling better? Is this a placebo effect? Since laying hands on animals can heal them, the Reiki (energy) healing couldn't be 'imaginary' (placebo) effect.

'Rei-Ki' means 'Universal energy' and the Reiki masters claim that they don't use their body energy to heal; they channel universal energy, which passes through their hands and enters the patient's body. They claim that some of the channeled energy remains in their bodies and goes mainly to fill the places with low energy, which are sick or going to be sick. In this way, by healing others they heal themselves.

Logically thinking, if energy is transferred to the patients during hand healing, the patients must gain some energy and the healers must lose some energy, for which they would compensate later on

*Corresponding author: Maria Kuman, Holistic Research Institute, 1414, Knoxville, USA, Tel: +1 8653094901; E-mail: holisticare1@gmail.com

Citation: Kuman M (2017) Measuring Reiki Healing - Mystery, Placebo or Real Energy Healing. J Altern Complement Integr Med 3: 022.

Received: July 05, 2016; Accepted: February 03, 2017; Published: February 17,2017 -this, was the initial hypothesis. It was difficult to believe that the hand healers channel universal energy during hand healing and heal themselves.

In order to check the hypothesis, a decision was made to measure Reiki healers and their patients. If the measured energy was electromagnetic, both electric and magnetic measurements should be able to give the desired information. Since magnetic measurements require shielding, only electric measurements were used on both the Reiki healers and their patients and the literature was checked if such measurements have been done before.

\section{Historic Background}

Dr. Herold Saxton Burr, Professor at the Yale University School of Medicine, first suggested in 1930s that diseases could be detected in the energy field of the body before the physical symptoms would appear. Also, he proposed in 1932 an electrodynamic theory of development [1]. In 1935 he published an article entitled "The electrodynamic theory of Life"with FSC Northrop as a co author [2]. In 1936, he published with CT Lane and LF Nims another article: A vacuum tube micro voltmeter for measurements of bioelectric phenomena [3]. In 1962 he wrote a book: The Nature of Man and the Meaning of Existence [4] and in 1973 he wrote another book: The Fields of Life [5].

Dr. John Zimerman [6], when working at the National Bureau of Standards, measured with SQUID people using the hand healing modality "Therapeutic Touch" He found pulsating biomagnetic field emitted from their hands with frequency $0.3-30 \mathrm{~Hz}$. He observed the following phenomena: when the right and left hemispheres of the brain become balanced and show alpha rhythm with frequency 7.8- 8 $\mathrm{Hz}$, he found that the electromagnetic emission of the healers' hands have the same frequency of pulsation (The alpha rhythm of the human brain with frequency $8-13 \mathrm{~Hz}$ is synchronized with the frequency 8-13 $\mathrm{Hz}$ of the electric field of the atmosphere and the frequency $8-13 \mathrm{~Hz}$ of the magnetic field of the earth).

\section{Body Energy and Acupuncture Meridians}

Energy balance of our body determines our health. The body energy circulates along a system of acupuncture meridians, each representing different organ on the surface of the skin. Each meridian is a chain of electrically conducting ellipses (called acupuncture points) oriented with their long axes along the chain (meridian). These conducting ellipses are imbedded in the semi conducting tissue of our body [7]. Electrical gradients determine the direction of the acupuncture meridians, which are usually vertical straight lines running upwards in the front side and downwards on the backside of the body.

Electric measurements were done on acupuncture meridians and it was found that if an organ is sick, its representing meridian on the surface of the skin is out of balance and certain point, called trigger point, usually has lower than normal electrical potential. When the trigger point is treated with a series of daily acupuncture treatments, one can see how the electrical potential of the treated point increases after each treatment. Simultaneously, the patient pain complains also decreases. When the electrical potential of the treated acupuncture point is back to normal, there are no more pain complains, and the disease will be cured. 
Dr. Hiroshi Motoyama (Japan) first measured the acupuncture meridians of Reiki healers and their patients before and after Reiki healing $[8,9]$. He found that both the patient and healer had different energy after the healing, but his data did not provide straight forward answer for one-way energy transfer from healer to patient.

Dr. Motoyama [8,9], found that after healing in some cases the energy of the Reiki healer's meridian for a particular organ was lower and in some cases his heart meridian had higher energy, but the results were not persistent. Either his measuring equipment did not have the needed sensitivity and accuracy, or the measured Reiki healers were not adequately trained (see the discussion). Obviously, the Reiki healers needed to be remeasured with the equipment with higher sensitivity and accuracy.

\section{Methods}

To measure the energy changes, which Reiki Healing creates in the body, noninvasive equipment with high sensitivity was built and patented (\#08/966,727). The measuring system consisted of: measuring equipment with one reference electrode hold in the left hand of the measured individual and certain number of specially designed measuring electrodes placed on the measured points (special paste was used to improve the contact). The system also included a system analyzer and a computer. Measurements were taken from both the patient and healer, before and after each Reike Healing.

Software was built explaining the trigger acupuncture points where the energy must be measured for: Heart (HT7), Solar plexus (CT12), Stomach (ST36), Colon (SI4), Body energy (TE6), Bladder (BL24 measured at the right and left sides of the backbone) and the point of energy union on top of the head Bai Huei (GV20). These most frequently used acupuncture points were measured to provide adequate information about the state of health or disease of the organs and the body as a whole. Measurements of other patients were also done along the backbone, which reflected the balance or imbalance of the vertebras and the neurons exiting between them.

\section{Results}

The high sensitivity energy meter was measuring subtle electrical characteristics of the human electromagnetic field. Reiki Master Mary Lou and her graduates were measured with this sensitive equipment and the results were consistent except on one student who was still in the process of learning. Her graduates were obviously well trained how to channel energy. Later, a lot more measurement were done on her students and their patients, studying the effect of crystals, effect of channeling, effect of colors, etc., and the results were consistent and meaningful.

Figures 1-4 reflect the measurements of four Reiki healers and their patients before (PRE) and after (POST) Reiki Healing sessions and nothing was known prior about the healers or their patients. Below are posted the figures (graphics) seen on the screen of the computer, which reflect our measurements.

The lower curve PRE reflects her measurements before the healing session and the top curve POST after it. From these curves one can see that her energy substantially increased after healing, which indicates that she did not use her energy to heal. Her higher energy after healing could mean only one thing that a third source of energy was involved. Thus, our measurements show that the claim of the Reiki healers that they channeled Universal energy seemed to be right.

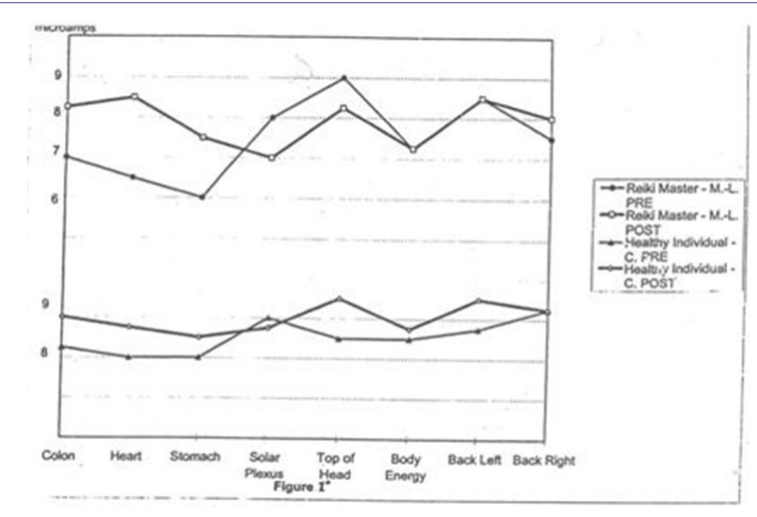

Figure 1: The measured energy balance of Reiki Master Mary Lou K. before (PRE) and after (POST) energy healing (top curves) and the measured energy balance of the treated person before (PRE) and after (POST) energy healing (lower curves).

The top two curves represents the energy balance of Reiki Master Mary Lou K. The lower curves represent the energy balance of the treated with energy persons.

The measurements also showed that the channeled energy indeed filled first the healers' organ with lowest energy, which would become sick. The low energy reading of the stomach and back of Reiki Master Mary Lou before the healing session (PRE) indicates digestion and spinal problems. Indeed, when asked after the measurements, she acknowledged the fact that she had food allergies and some back pain.

As one can see from Mary Lou's top (POST) curve in figure 1, after the Reiki Healing session the energy of her stomach and back were much higher, this means that her stomach and back were better. This indicates that the hand healer Mary Lou benefited from the healing session as well. Obviously, she was having only minor health complains because she was doing a lot of hand healing, which was improving her energy balance.

Perfect health means perfect energy balance represented graphically by a strait horizontal line [4]. From the graph, it is clear that after healing the energy balance of Reiki Master Mary Lou had improved, but it was still far from a straight line. If you look at the energy balance of the person she treated (Charlie), you will see her imbalance imprinted on his energy balance. This explains why the original teaching of Usui was: "Balance yourself first before doing any Reiki healing!"

The lower two curves on figure 1 reflect the energy balance of Charlie, who was treated by Reiki Master Mary Lou. One can see from his energy balance before the treatment (bottom curve PRE), which is close to a straight horizontal line, that he was a healthy individual [4]. After Reiki Master Mary Lou treated him, his energy balance (see top curve POST) is even closer to a straight line [4], which means his health has improved. Therefore, even a healthy individual can benefit from a Reiki treatment, which will improve his energy balance and thus improve his health. The Reiki healers (\#2 and \#3) were graduates of Reiki Master Mary Lou and Reiki Healer \#4 was her student.

The measurements of Norm (Fig 2) after he did hand healing (curve POST) show increase of the energy of his stomach and lower back, which confirms his statement after the measurements that his stomach and lower back feel better after each hand healing. Norm explained that he has had lower back pain for a long time; he even had back surgery, which helped temporarily, but after a while the pain came back. He said Reiki healing reduces his pain - when healing others, he heal himself [10]. 


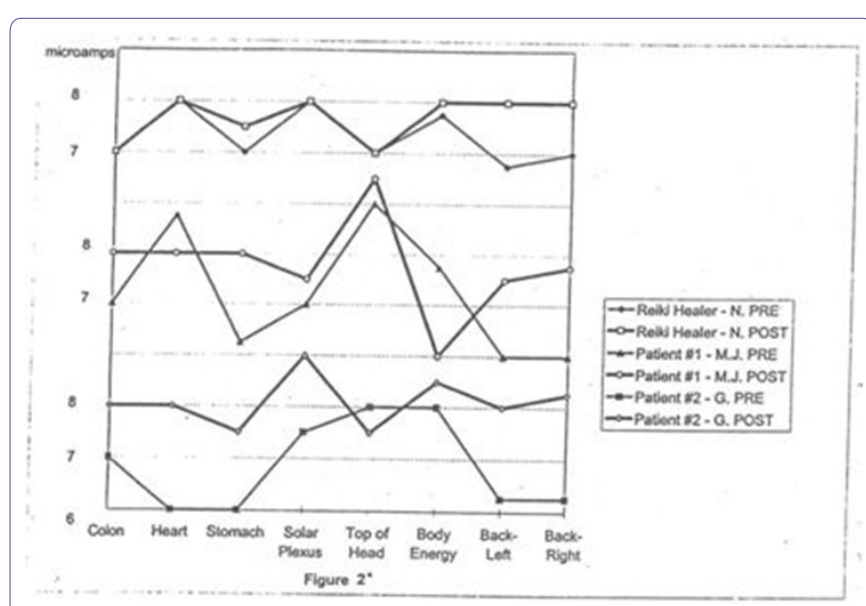

Figure 2: The measured energy balance of graduated Reiki Healer Norm K. before (PRE) and after (POST) energy healing (top curves) and the measured energy balance of the treated by him persons before (PRE) and after (POST) energy healing (lower curves).

The top two curves represents the energy balance of Reiki Healer Norm K, a graduate of Reiki Master Mary Lou. The low readings of his stomach and back before the healing session (curve PRE) indicate digestion and low back problems. Later conversation with him confirmed that he has digestion problem. It was recently found that he has gluten intolerance and gluten free diet helped him feel better. He said doing Reiki healing helps him feel better, too.

The lower curves represent the energy balance of the treated with energy persons.

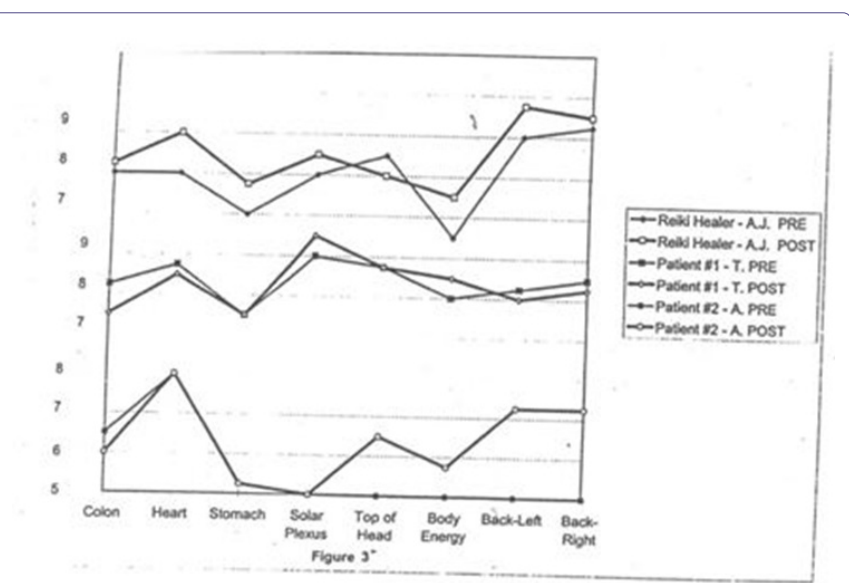

Figure 3: The measured energy balance of graduated Reiki Healer Ann J. before (PRE) and after (POST) energy healing (top curves) and the measured energy balance of the treated by her persons before (PRE) and after (POST) energy healing (lower curves).

The top two curves represents the energy balance of the Reiki Healer Ann J, a graduate of Reiki Master Mary Lou. Bottom curve (PRE) reflects her measurements before the healing session and top curve (POST) after it. The low reading of her body energy point TE6 indicates exhaustion, while the low energy reading of her stomach point indicates digestion problems. After the measurements, she acknowledged that before the healing session began she felt very exhausted. For this reason, her first patient $\mathrm{T}$ did not show substantial improvement.

The lower curves represent the energy balance of the treated with energy persons

Both Norm's patients MJ and G also had low energy readings of stomach and back (low curves PRE) and both of them showed improvement after Reiki healing (see top curves POST). However, after healing the POST energy balance of patient $\mathrm{G}$ is closer to a straight line (perfect health balance), the health POST curve of MJ is still very much out of balance regardless of the substantially improved energy readings of her stomach and back.

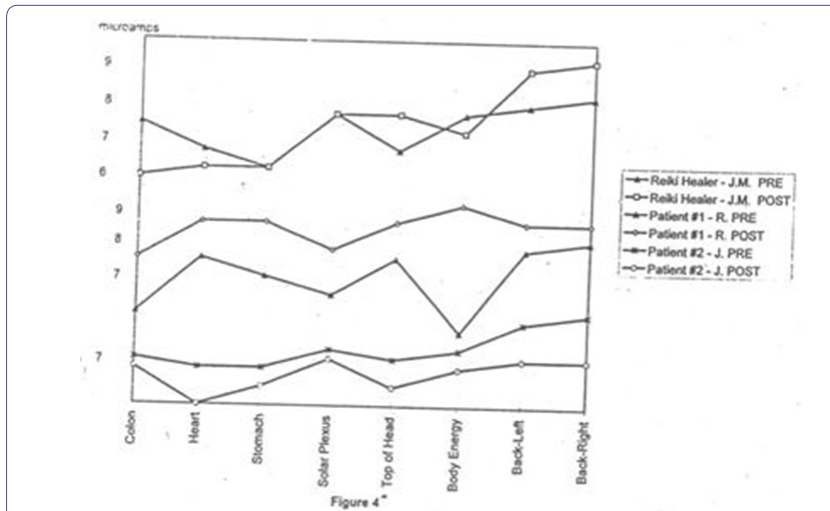

Figure 4: The measured energy balance of Reiki student Jim M. before (PRE) and after (POST) energy healing (top curves) and the measured energy balance of the treated by him persons before (PRE) and after (POST) energy healing (lower curves).

The top two curves represents the energy balance of the Reiki student Jim, who was still studding Reiki with Reiki Master Mary Lou. The curve (PRE) reflects his measurements before the healing session and the curve (POST) after it.

The lower curves represent the energy balance of the treated with energy persons

The reason for this was: the patient MJ had serious pathological problems: obesity, hormonal imbalance, and a lot of health complaints. She obviously needed a lot of healing for substantial improvement [10].

However, Ann obviously felt recharged after the first healing session, as her POST curve shows higher energy reading of her body energy point TE 6 and an increase of her total body energy and balance, i.e., her energy balance was closer to a straight line [10]. The effect of her second Reiki healing (top of the bottom curves on Figure 3) was much stronger.

This second patient had flu and felt very miserable, which explains very low energy readings before the treatment. After the healing session, however, her energy substantially improved and she said she felt much better.

Comparing the energy curves of this Reiki student before and after healing session with the energy curves of the rest of the Reiki healers, who were Reiki graduates, one can see that after healing their energetic balance improved (becoming closer to a straight line [10]), the energy balance of Reiki student Jim was worse after the 'healing. The energy curves of the two patients he treated are plotted under his curves on figure 4. From these curves it becomes obvious that 'healer' Jim picked the energy imbalance of his patient \# 1 (middle curves), which made him more imbalanced. As a result of this, the second patient he treated had lower energy after the treatment and was more imbalanced.

When the graphics were plotted (at the beginning our system was not computerized yet), I called Reiki Master Mary Lou and told her that there was something wrong with Jim's 'healing. She said, she already knows about this. After the healing session was over, Jim went to her complaining about inflicted back pain and she had to treat him for this. Once he picked the energy pattern of his patient \#1, he picked his health troubles and pain.

\section{Discussion and Conclusion}

According to Motoyama, the measurements did not detect any energy decrease in organs of the healer after healing $[8,9]$. In contrary, the total energy of the healers increased after healing and the increase 
was maximal in the organs with low energy, who are sick or going to be sick, which leads to the confirmation of Reiki Masters statement i.e., by healing others they heal themselves.

The difference between our measurements and that of Dr. Motoyama $[8,9]$ could mean that either he used measuring equipment with not enough sensitivity, or he measured not adequately trained hand healers. I measured therapeutic touch healers (another form of hand healing) and the results were not as consistent as the results from measuring the graduates of Reiki Master Mary Lou.

The measurements didn't support my initial hypothesis that Reiki healing probably works on the principle of give and take, which meant that if the patients were gaining some energy, the healers would be losing some energy. The first obvious thing in the data curves (Figures 1-3) is, both the patient and healer energetically benefit from the Reiki healing session. The healers' energies are definitely higher and their energy balance is better (closer to a straight line, which means perfect health) [10].

There could be only one explanation to the data curves of the Reiki healers - they are not using their own body energy to heal. The fact that their energies have increased after healing and their energetic balance has improved, means there is a third source of energy, from which they draw.

Since the Reiki healers claim that they channel universal cosmic energy to heal, and the electrical energy measured at the top of their

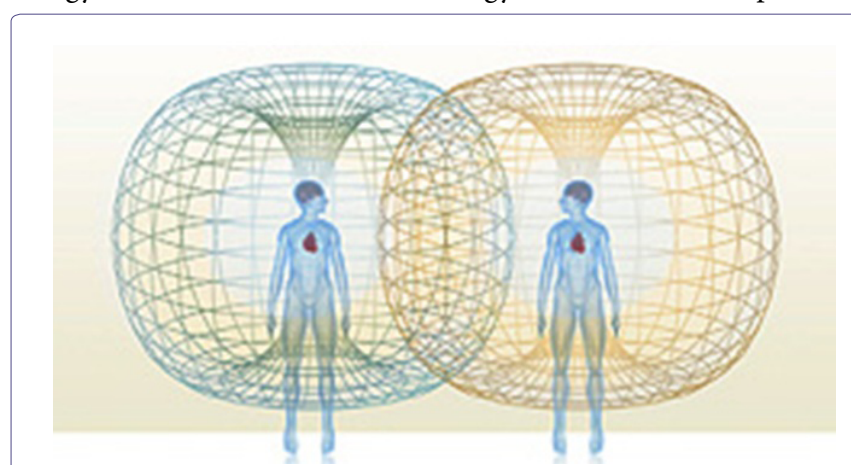

Figure 5: Donut-shaped human electromagnetic field (by the Heart Math Institute in California).

heads (GV20) increased after each healing, it seems that they indeed channel to their patients electric energy from the atmosphere, which is formulated in their teaching as cosmic (Universal) energy. Only by accepting this, can we explain the fact that when channeling this Universal energy they benefit from it too.

Measurements of the human electromagnetic field (by the Heart Math Institute in California) shows that it has the form of a donut with a very narrow hole in the middle with one opening on top of the head and another at the tailbone (Figure 5). The donut-shaped human field is spinning (measured by Shkatov (Russia) [11]) and the top opening is a vortex, through which the human body can suck down electrical energy from the atmosphere [11].

The spinning donut shaped human field has opening toward the earth, which is anti vortex. Through it the human body can suck upward magnetic energy from the earth (Interestingly, the electromagnetic fields of our planet and Sun have the same torus (donut) shape).

Our measurements also showed that Reiki Healing could be done wrong, if the healer does not follow exactly the recommendations of his Reiki master. Instead of channeling universal cosmic energy, the 'healer' can pick the energy imbalance of his patient (Figure 4) and his patient's troubles and pain. This was the case with Jim (Figure 4). $\mathrm{He}$ needed treatment from his Reiki Master to get rid of the inflicted pain.

This means that the Reiki healers must strictly follow the channeling and healing procedure recommended by their Reiki master. This could be one of the reasons why Dr. Motoyama [8,9] could not get consistent repeatable results.

Our measurements also showed why the recommendations of the ancient Reiki Masters: "balance your energy first before doing any hand healing" must always be followed. Looking at the energy curves of the three hand healers presented in figures 1-3, one could see that when the healer was not balanced, his imbalance became imprinted on the energy balance of the treated person.

The greatest advantage of energy healing is: it can be used for prevention. Energy imbalance precedes the symptoms of each disease and if the energy is balanced through energy healing with hands or acupuncture, the symptoms of the disease would never appear, and the disease would be prevented.

\section{Acknowledgement}

The author acknowledges the organizing skill of Reiki Master Mary Lou Kuneman and expresses gratitude for her help in organizing the measurements.

\section{References}

1. Burr HS (1932) An electro-dynamic theory of development suggested by studies of proliferation rates in the brain of Amblystoma. The Journal of Comparative Neurology 56: 347-371.

2. Burr HS, Northrop FS C (1935) The Electro-Dynamic Theory of Life. The Quarterly Review of Biology 10: 322-333.

3. Burr HS, Lane CT, Nims LF (1936) A Vacuum Tube Micro-voltmeter for the Measurement of Bio-electric Phenomena. Yale J Biol Med 9: 65-76.

4. Burr HS (1962) The Nature of Man and the Meaning of Existence. Springfield, IL, USA.

5. Burr HS (1972) The fields of life. Our links with the universe, Ballantine Books, New York, USA.

6. Williamson SJ (1989) Advances in Biomagnetism. Plenum Press, Berlin, Germany.

7. Kuman M (1997) Modern Aspects of Ancient Acupuncture-Acupuncture without Needles. Holistic Health and Happiness, Knoxville, Tennessee, USA.

8. Motoyama H (1979) The Functional Relationship between Yoga Asanas and Acupuncture Meridians, IARP, Tokyo, Japan.

9. Motoyama H (1981) A Biophysical Elucidation of the Meridian and Ki Energy, What Is Ki Energy and How Does It Flow. Research for Religion and Parapsychology 7: 1.

10. Kuman M (2000) Listen and Talk to Your Body and Soul. Holistic Health and Happiness, Knoxville, Tennessee, USA.

11. Kuman M (2017) Dynamic of Creation and Functioning of Galaxies, Black Holes, and Man, Health and Happiness Books. 